\title{
Wissenschaftskommunikation für Unternehmen: Wieso, weshalb, warum?
}

\author{
Patricia Piekenbrock
}

Können Medien, Politik und Wirtschaft noch punkten, wenn große Teile der Bevölkerung wissenschaftlichen Erkenntnissen misstrauen? Haben Industrieunternehmen in diesem Szenario überhaupt eine Chance, ihre Ergebnisse aus Forschung und Entwicklung glaubwürdig und überzeugend zu kommunizieren? Und wie sorgfältig trennen Unternehmen Wissenschaftskommunikation und Marketing?

\section{Sind wir heute desillusioniert?}

Gesellschaftskritik ist jeder Epoche inhärent. Diese Kritik ist auch notwendig, will man neuen Ideen Raum verschaffen und Kreativität für Veränderungen entstehen lassen. Solche Vorgänge schüren jedoch auch Ängste und säen Zweifel, wenn die Befürchtung besteht, sie könnten Verschlechterungen für bestimmte Zielgruppen nach sich ziehen. In der ersten Hälfte des 19. Jahrhunderts zeichnete Honoré de Balzac in seinem dreiteiligen Roman „Illusions perdues“ ein düsteres Gesellschaftsbild der Pariser Medienlandschaft: ein Milieu ehrgeiziger Schriftsteller, käuflicher Journalisten, korrupter Massenblätter, sensationsheischender Leser und erpressbarer Politiker.

Solch moralischer Sumpf wirft existenzielle Fragen auf und lässt Hoffnungen auf einen Diskurs zwischen Volk und Herrscher - heute würden wir

P. Piekenbrock $(\square)$

Kommunikation für Wissenschaft, Forschung und Technologie, Berlin,

Deutschland

E-Mail: info@piekenbrock-kommunikation.de 
vielleicht sagen: Gesellschaft, Politik und Wirtschaft - dahinschmelzen. Es fällt uns nicht schwer, Analogien im Hier und Jetzt zu finden. Die Bürger misstrauen Medien, Politik, staatlichen Institutionen und Unternehmen. Teils ist dieses Misstrauen berechtigt, oft beruht es auf problematischem Halbwissen oder wird von bestimmten Interessens- und Lobbygruppen geschürt. Sogenannte alternative Fakten wirken in ihrer Einfachheit verführerisch, täuschen sie doch eine leicht verständliche Problemlösung vor. Glaubwürdigkeit kann kaum vermittelt werden, insbesondere wenn der Begriff „Lügenpresse“ im Raum steht.

\section{Interessen versus Vertrauen?}

Regieren heute also die Medienkonsumenten durch ihr Klickverhalten, leiden Wissenschaftler unter blinder Publikationswut, stellen Verlage geschönte Informationen bereit und lancieren Unternehmen, was und wo sie wollen? Beschäftigt man sich im Feld der Wissenschaftskommunikation einzig und allein damit, wer von wem mit wessen Geld gekauft wird, gewinnen materielle oder machtpolitische Abhängigkeiten die Oberhand über Inhalte und Botschaften. Ein solcher Ansatz ist bereits in seinem Kern problematisch.

Für die moderne Unternehmenskommunikation stellt sich diese Situation als Antinomie dar. Einerseits ist sie darauf angewiesen, Vertrauen zu schaffen - ein kostbares Gut, auf dem die zarte Pflanze der Reputation gedeiht. Andererseits möchte sie Produkte, Technologien oder Unternehmensperspektiven ins gewünschte rechte Licht rücken. Entlastet beispielsweise ein neu entwickelter Industrieroboter einen von Rückenschmerzen geplagten Werker von körperlich anstrengenden Tätigkeiten oder nimmt er ihm seinen Arbeitsplatz weg? Die Antwort darauf ist weder einfach noch eindeutig: Sie ist individuell zu geben. Wissenschaftskommunikation in der Industrie ist also stets ein Handwerk, das sich die Frage nach der eigenen ethischen Gesinnung stellen muss.

Gewiss, letztlich vertritt jeder Mensch, jedes Individuum seine eigenen Interessen: in beruflicher, sozialer und privater Hinsicht. Wissenschaftler, Journalisten, Kommunikatoren, Politiker oder Manager sind davon nicht ausgenommen, auch wenn das nicht alle freimütig einräumen. So ist auch ein ambitionierter, wertneutral arbeitender Wissenschaftsjournalist darauf angewiesen, seine Beiträge in der Redaktionsrunde thematisch durchzusetzen und innerhalb eines angemessenen Zeitfensters zu erstellen. Der Preis für erschüttertes Vertrauen in Wissenschaft, Medien und Unternehmen ist hoch. Menschen fühlen sich verunsichert oder getäuscht. In einer Atmosphäre des Misstrauens gedeiht wenig Positives, im Gegenteil: Die Summe gesamtgesellschaftlichen Vertrauens verschiebt und verteilt sich in temporär 
glaubwürdige, diffuse und volatile Richtungen. Deshalb gilt für alle Akteure, eine vertrauensvolle Atmosphäre zu schaffen.

Auch wenn der Journalismus - ausgestattet mit dem moralischen Rüstzeug des Pressekodex - das deutlich schärfere Schwert in Sachen Reflexion und Kritik zu führen hat, darf sich eine professionelle Wissenschaftskommunikation, die im Namen und Auftrag eines Unternehmens geführt wird, hiervon nicht absetzen. Sie muss die oben erwähnte Gefahr eines Vertrauensverlusts erkennen und unter Umständen auch gegenüber ihrem Auftraggeber zum Ausdruck bringen. Oftmals zeigt sich diese Problematik bei börsennotierten Unternehmen, wenn Manager einem enormen internen und externen Erwartungsdruck mit Blick auf kurzfristige wirtschaftliche Ergebnisse ausgesetzt sind.

\section{Sprechen wir dieselbe Sprache?}

Laut Zahlen des Statistischen Bundesamts aus dem Jahr 2017 verfügen lediglich 17 Prozent der Bevölkerung in Deutschland über einen akademischen Abschluss, vier Prozent können überhaupt keinen allgemeinen Schulabschluss vorweisen. Vielen Menschen erschließt sich das Reflexionsniveau der Wissenschaft nicht unmittelbar und manche kämpfen beim Lesen eines Feuilletonartikels mit Verständnisschwierigkeiten. Es fällt schwer, jemandem zu vertrauen, dessen Worte wir nicht verstehen können. Sprache ist und bleibt das wichtigste Transportmittel aller Inhalte, visuelle Medien mit eingeschlossen.

\section{Lebenswelten, Zeitnot und Sprache}

Wenn das tagtäglich zu organisierende Dasein von Beruf, Familie, Freunden und Haushalt dominiert wird, entsteht Zeitdruck. Die Schnelllebigkeit und das Effizienzgebaren unserer Zeit tragen das Ihre dazu bei. So sinkt die Bereitschaft des Einzelnen, sich mit Texten auseinanderzusetzen. Besteht dennoch ein gewisses Leseinteresse, geht es oft mit der Sehnsucht nach Einfachheit und dem Wunsch nach gedanklicher Erholung einher. So wirkt die Beschleunigung auf unser wichtigstes Kommunikationsinstrument: Das Niveau der Sprache wandelt sich. Mangelnde Ausdrucksfähigkeit, einfacher Wortschatz, Grammatikabbau und orthografische Schwierigkeiten sind nur einige der drastischen Ausblühungen. Die Sprache wiederum beeinflusst Gedankenwelten, Anschauungen und Haltungen.

\section{Chancen für Unternehmen?}

Unternehmen eröffnen sich heute vielfältige Möglichkeiten: Ausgestattet mit Budgets, von denen Zeitungen oder Magazine nur träumen können, 
betreiben sie Wissenschafts- und Technologiekommunikation häufig auf medial höchstem Niveau. Ob durch Freelancer oder Agenturen redaktionell begleitet, entstehen im Bereich Text und Grafik, Film und neue Medien, Kultur- und Sozialsponsoring innovative PR-Produkte, die nicht selten Trends setzen und ästhetisch State of the Art sind.

Im Gegensatz zu Wissenschaftlern können und dürfen sie - ohne um ihre Reputation bangen zu müssen - eine einfache, angemessene und verständliche Sprache anwenden und mit emotional aufgeladenen Bildwelten arbeiten. Wissenschaftlichkeit und Kompetenz der Unternehmen bleiben davon unberührt. Im Gegenteil: Wenn sie sich an ihre Zielgruppen und -personen wenden, etwa Meinungsbildner, Journalisten, Bildungsträger, Politiker oder Kunden, sind Unternehmen frei in der Wahl des Publikationszeitpunkts und des Kommunikationsformats. Aus ihrem Rucksack zaubern sie professionell aufbereitetes Informationsmaterial, dessen Verwendung Medien kaum ablehnen können. Dies wird besonders deutlich, wenn man einen intensiven Blick auf zukunftsweisende Themenfelder der Medizin, Robotik, künstlichen Intelligenz, Bio- und Informationstechnologien wirft.

\section{Informations- und Wissensquellen}

Als Vorteil können Unternehmenskommunikatoren zudem namentlich die Quellenfrage für sich verbuchen - dieser Punkt wird in der Öffentlichkeit selten wahrgenommen. Jeder von uns empfängt eine Flut von Informationen aus dem Internet, auf dem Smartphone und auf öffentlichen Bildschirmen; meist sogar zeitgleich. Allerdings bleibt häufig verborgen, woher die Daten und Nachrichten stammen. Welcher Information kann ich vertrauen, an welcher Stelle sollte ich hinterfragen? Industrieunternehmen verfahren beim Versand ihrer Botschaften in aller Regel als Absender transparent, denn an dieser Stelle wird ihre Reputation berührt. Der Rezipient vermag nachzuvollziehen, dass es sich um eine Einrichtung mit wirtschaftlicher Ausrichtung handelt, und hat zudem die Möglichkeit der Kontaktaufnahme. Der Informationsgehalt wird an dieser Stelle - gerade vor dem heterogenen und teilweise undurchsichtigen Hintergrund der heutigen Medienlandschaft nicht geschmälert.

Dazu kommt, dass Akteure im Wissenschafts- und Technologiesektor häufig nicht mehr klar voneinander zu trennen sind. Firmen forschen in Verbünden und kooperieren untereinander, sie arbeiten mit Hochschulen, Instituten und Bildungseinrichtungen zusammen. Feste und freie Mitarbeiter, Praktikanten, Studenten, aber auch Führungskräfte werden temporär ausgetauscht bzw. wechseln ihren Arbeitsplatz. Kommunikatoren in forschenden Unternehmen stehen dabei im kontinuierlichen Austausch 
mit den eigenen Wissenschaftlern. Sie können Entwicklungen mit ihren erwartungsvollen Hochs und problematischen Tiefs verfolgen und entsprechend kommunizieren. Ein solch persönlicher, naher und direkter Zugang bleibt Medienvertretern häufig verwehrt.

\section{Wer soll was lesen?}

Zielgruppenanalyse ist ein bewährtes Instrument der Unternehmen, einzelne Kommunikationsmaßnahmen auszurichten. Eine differenzierte Beschäftigung bzw. ein Hineinversetzen in den künftigen Rezipienten zahlt sich aus: Die Adressaten werden Informationen nur wahrnehmen, wenn sie in der für sie angemessenen Sprache und im geeigneten Format aufbereitet sind. Selbst komplexe und erklärungsintensive Botschaften lassen sich verständlich, angemessen und korrekt transportieren - ob in multimedialer Kommunikation, in den sozialen Medien oder im persönlichen Dialog.

„Ihren“ Lesestoff werden Kinder, Jugendliche, Bildungseinrichtungen, aktive und künftige Mitarbeiter, Wettbewerber, Politiker, Führungskräfte und Endkunden erkennen und wahrnehmen. Ein klassisches Kinderbuch zu einem technologischen Thema, das multimedial aufbereitet in mehreren Landessprachen angeboten wird, kann zum Beispiel mehrfache Effekte erzielen: Der Manager eines indischen Unternehmens etwa wird wahrscheinlich einen Blick in das ihm überreichte, ansprechend gestaltete Buch werfen und es durchblättern, bevor er es seinem Sohn übergibt, der sich später um eine Praktikumsstelle bei dem absendenden Unternehmen bewirbt. Die Botschaft ist angekommen.

\section{Verständlichkeit schafft Vertrauen}

Unternehmen, die Wissenschaftskommunikation in eigener Sache betreiben, sollten sich bewusst sein: Mit den sich eröffnenden Chancen gehen auch Risiken einher. Eine Top-down-Kultur, die heute manchen Managementhierarchien noch inhärent ist, kann im Bereich der Wissenschaftskommunikation versagen. Ein durch Marketingroutinen geprägtes Sprachverständnis birgt die Gefahr, dass inhaltlich und stilistisch an den gewünschten Zielgruppen vorbeigeschrieben und kein Mehrwert generiert wird. Es bedarf einer intensiven und professionell angeleiteten Analyse, nicht nur welche Medien für welche Botschaften geeignet und welche Informationen für Kunden relevant sind, sondern auch welche Schranken der Werblichkeit gesetzt werden sollten. Insbesondere wenn juristische, ethische, psychologische oder politische Belange berührt werden, sollte sich ein Wissenschaftskommunikator stets auch als Korrektiv des Gewünschten betrachten. 
Selbstverständlich besteht seine Aufgabe primär darin, Produkte, Leistungen und die positiven Aspekte von Sachverhalten zu betonen. Aber zugleich muss er möglichen Schaden für sein Unternehmen abwenden, falls Innovationen überzeichnet oder Gefahrenpotenziale nicht ausreichend beziehungsweise adäquat zur Sprache kommen.

Verständlichkeit fördert Vertrauen, durch Vertrauen wachsen Interesse und Engagement. Und die Erfahrungen der letzten Jahre haben gezeigt: Die bundesweiten Unternehmen im Wissenschafts- und Technologiebereich haben beste Chancen, hier differenziert anzusetzen. Über Leistungen und Innovationen muss berichtet, thematische Einordnungen müssen gefunden und gesellschaftliche Lösungen erarbeitet werden. Gerade eine professionelle Unternehmenskommunikation kann dazu beitragen, neben der existierenden Konsumkultur schrittweise eine offenere Frage- und Diskussionskultur zu etablieren. Gesellschaftliche Partizipation bedeutet auch, dass der einzelne Mensch seine persönlichen Fähigkeiten, Voraussetzungen, Erfahrungen und Gegebenheiten in den Diskurs einbringen darf. Einbinden statt abgrenzen. Viele Unternehmen haben dies bereits verstanden, manche suchen noch nach der rechten Sprache. Doch diese Problematik ist in der Regel keine der Medien oder ihrer Kunden.

Patricia Piekenbrock absolvierte ihren Studienabschluss als Diplom-Physikerin in Heidelberg, zusätzlich erwarb sie den Master of Advanced Studies in Journalism an der Donau-Universität Krems in Österreich. Als freie Kommunikationsberaterin betreut sie Kunden aus Industrie, Wissenschaft und Stiftungswesen. Ihre Schwerpunkte sind dabei fachübergreifende Themenfelder wie Bionik, Automation und Robotik. Vor ihrer Selbstständigkeit war sie als Konzernpressesprecherin und Redakteurin für Technologiethemen der Daimler AG tätig. 
Open Access Dieses Kapitel wird unter der Creative Commons Namensnennung 4.0 International Lizenz (http://creativecommons.org/licenses/by/4.0/deed.de) veröffentlicht, welche die Nutzung, Vervielfältigung, Bearbeitung, Verbreitung und Wiedergabe in jeglichem Medium und Format erlaubt, sofern Sie den/die ursprünglichen Autor(en) und die Quelle ordnungsgemäß nennen, einen Link zur Creative Commons Lizenz beifügen und angeben, ob Änderungen vorgenommen wurden.

Die in diesem Kapitel enthaltenen Bilder und sonstiges Drittmaterial unterliegen ebenfalls der genannten Creative Commons Lizenz, sofern sich aus der Abbildungslegende nichts anderes ergibt. Sofern das betreffende Material nicht unter der genannten Creative Commons Lizenz steht und die betreffende Handlung nicht nach gesetzlichen Vorschriften erlaubt ist, ist für die oben aufgeführten Weiterverwendungen des Materials die Einwilligung des jeweiligen Rechteinhabers einzuholen. 